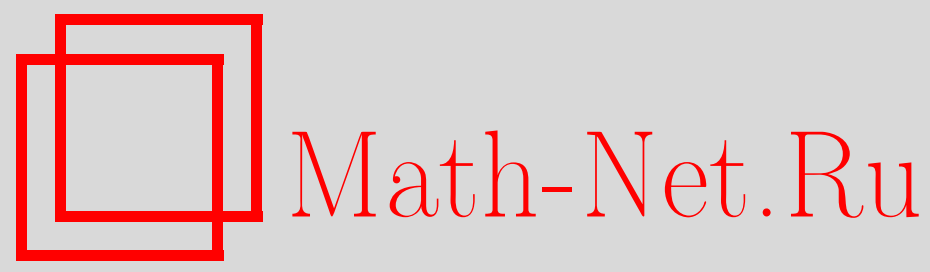

П. В. Филевич, Об оценке величины исключительного множества в лемме о логарифмической производной, Матем. заметки, 2000, том 67, выпуск 4, 603-607

DOI: https://doi.org/10.4213/mzm875

Использование Общероссийского математического портала Math-Net.Ru подразумевает, что вы прочитали и согласны с пользовательским соглашением http://www . mathnet.ru/rus/agreement

Параметры загрузки:

IP: 3.85 .183 .62

26 апреля 2023 г., 04:35:38






\section{ОБ ОЦЕНКЕ ВЕЛИЧИНЫ ИСКЛЮЧИТЕЛЬНОГО МНОЖЕСТВА В ЛЕММЕ О ЛОГАРИФМИЧЕСКОЙ ПРОИЗВОДНОЙ}

\section{П. В. Филевич}

В работе получена точная оценка величины исключительного множества в лемме о логарифмической производной.

Библиографиял: 6 названий.

$\mathbf{1}^{\circ}$. Как известно, ряд соотношений между характеристиками мероморфных и,в частности, целых функций выполняются вне некоторого исключительного множества $E \subset$ $[1 ;+\infty)$, о котором известно чаще всего, что оно имеет конечную меру или конечную логарифмическую меру. Такими являются, например, соотношения между максимумом модуля $M_{f}(r)=\max \{|f(z)|:|z|=r\}$, максимальным членом $\mu_{f}(r)=\max \left\{\left|f^{(n)}(0) / n !\right|\right.$ $\left.\times r^{n}: n \geqslant 0\right\}$ и центральньм индексом $\nu_{f}(r)=\max \left\{n \geqslant 0:\left|f^{(n)}(0) / n !\right| r^{n}=\mu_{f}(r)\right\}$ в известных теоремах Вимана-Валирона для целых функций (см., например, [1], [2, c. 17-29]), соотношения между характеристиками Неванлинны в лемме о логарифмической производной $[3$, c. 122$]$ и второй основной теореме Неванлинны о распределении значений [3, с. 127$]$ для мероморфных функций.

Из слов О.Б. Скаскива автору известно, что в устном разговоре с ним И. В. Островский ставил вопрос (открытый и сейчас) о возможности получения точной оценки величины исключительного множества $E(\varepsilon, f)$ в следующем неравенстве Вимана-Валирона:

$$
M_{f}(r) \leqslant \mu_{f}(r) \ln ^{1 / 2+\varepsilon} \mu_{f}(r) \quad(r \geqslant 1, \quad r \notin E(\varepsilon, f)) .
$$

(Классическая оценка такая: $\int_{E(\varepsilon, f)} r^{-1} d r<+\infty$, т. е. $E(\varepsilon, f)$ имеет конечную логарифмическую меру для любого $\varepsilon>0$ и любой целой функции $f$.) Понятно, что аналогичные вопросы можно рассматривать и по отношению к исключительным множествам в других соотношениях. В этой работеполучена точная оценкавеличины исключительного множества в лемме о логарифмической производной.

$\mathbf{2}^{\circ}$. Пусть $H-$ класс положительных, непрерьвных, возрастающих к $+\infty$ на $[1 ;+\infty)$ действительных функций, а $L$ - подкласс вьпуклых относительно $\ln r$ на $[0 ;+\infty)$ функций из $H$. Как обычно,

$$
\rho(h)=\varlimsup_{r \rightarrow+\infty} \frac{\ln h(r)}{\ln r}
$$

- порядок функции $h \in H(0 \leqslant \rho(f) \leqslant+\infty)$. Порядок мероморфной функции $f$ - это порядок ее характеристической функции Неванлинны $T_{f} \in L$. 
Пусть $m_{f}(r)=(2 \pi)^{-1} \int_{0}^{2 \pi} \ln ^{+}\left|f\left(r e^{i \varphi}\right)\right| d \varphi-$ характеристическая функция приближения мероморфной функции $f$ к $\infty$. Тогда по лемме о логарифмической производной $[3$, c. 122] для любой мероморфной функции $f$ вьполняется оценка

$$
m_{f^{\prime} / f}(r)=O\left(\ln T_{f}(r)+\ln r\right) \quad(r \rightarrow+\infty, \quad r \notin E(f)),
$$

где $E(f)$ - исключительное множество, отсутствующее в (1) для функций конечного порядка, т. е. для таких функций $m_{f^{\prime} / f}(r)=O(\ln r)(r \rightarrow+\infty)$.

Хейман [4] (см. также [3, с. 123]), опровергая известное предположение Неванлинны, показал, что для функций бесконечного порядка исключительное множество в соотношении (1), вообще говоря, существует. В связи с этим вопрос о получении точной оценки величины исключительного множества выглядит достаточно естественным. Лучшая из известных оценок приведена в [2, с. 37]: для любой мероморфной функции $f$ множество $E(f)$ в соотношении (1) можно подобрать так, что

$$
\int_{E(f)} r^{n-1} d r<+\infty, \quad n \geqslant 0 .
$$

Следующая теорема указывает на невозможность дальнейшего улучшения этой оценки.

ТЕОрема 1. Пусть $h \in H$. Тогда для того чтобы для любой мероморфной функиии $f$ существовало множество $E(f) \subset[1 ;+\infty)$ такое, что одновременно выполняются соотношения (1) $u \int_{E(f)} h(r) d r<+\infty$, необходимо и достаточно, чтобы $\rho(h)<+\infty$.

Достаточность условия $\rho(h)<+\infty$ следует из оценки (2). Необходимость получим из следующего утверждения.

ТЕорема 2. Пусть $h \in H, \rho(h)=+\infty$. Тогда существует иелая функиия $f$ такая, что:

1) $\nu_{f}(r) \leqslant h(r)\left(r \geqslant r_{0}\right)$

2) $M_{f}(r) \leqslant(2+o(1)) \mu_{f}(r)(r \rightarrow+\infty)$;

3) для мнохсества $E_{1}(\varepsilon, f)=\left\{r>1: T_{f^{\prime}}(r)>(1+\varepsilon) T_{f}(r)\right\}$ при некотором $\varepsilon>0$ выполнено равенство $\int_{E_{1}(\varepsilon, f)} h(r) d r=+\infty$.

Доказательство теоремы 2 будет приведено ниже. Сначала покажем, как из теоремы 2 получить необходимость условия $\rho(h)<+\infty$ в теореме 1 . Пусть $h \in H, \rho(h)=+\infty$, a $f$ - целая функция, обладающая свойством 3) из теоремы 2 . Если теперь предположить, что для функции $f$ соотношение $(1)$ вьполняется с множеством $E(f)$, для которого $\int_{E(f)} h(r) d r<+\infty$, то такая же оценка вьполняется и для множества $E_{2}(\varepsilon, f)=\{r>1$ : $\left.m_{f^{\prime} / f}(r)>\varepsilon T(r, f)\right\}$ при любом $\varepsilon>0$. (Это следует из того, что функция $f$, как легко видеть, является трансцендентной, а для таких функций $T_{f}(r) / \ln r \rightarrow+\infty$ при $r \rightarrow+\infty$.) Далее, так как

$$
T_{f^{\prime}}(r)=m_{f^{\prime}}(r) \leqslant m_{f}(r)+m_{f^{\prime} / f}(r)=T_{f}(r)+m_{f^{\prime} / f}(r)
$$

то множество $E_{1}(\varepsilon, f)$ содержится в множестве $E_{2}(\varepsilon, f)$, т. е. $\int_{E_{1}(\varepsilon, f)} h(r) d r<+\infty$. Получаем противоречие, поэтому в предположении справедливости теоремы 2 теорема 1 доказана. 
Отметим еще одно следствие из теоремы 2. Как известно, рост мероморфной функции $f$ часто связьвают с ростом ее характеристики $T_{f} \in L$ (это видно, например, из определения порядка мероморфной функции). Обозначим для любой $l \in L$ через $M(l)$ множество мероморфных функций $f$ таких, что $T_{f}(r) \leqslant l(r)\left(r \geqslant r_{1}(f)\right)$. Главньй вьвод из следующей теоремы состоит в том, что существуют мероморфные функции бесконечного порядка и сколь угодно медленного роста, для которых не упоминать об исключительном множестве в соотношении (1) нельзя.

ТЕОРема 3. Пусть $l \in L$. Для того чтобы для любой $f \in M(l)$ выполнялось соотношение

$$
m_{f^{\prime} / f}(r)=O\left(\ln T_{f}(r)+\ln r\right) \quad(r \rightarrow+\infty)
$$

необходимо и достаточно, чтобы $\rho(l)<+\infty$.

ДокАЗАТЕЛЬСтво. Достаточность условия $\rho(l)<+\infty$ в теореме 3 очевидна, поскольку тогда каждая функция $f \in M(l)$ имеет конечньй порядок. Докажем необходимость.

Пусть $l \in L, \rho(l)=+\infty, k(r)=r l^{\prime}(r)(r \geqslant 0)$, т.е. $k(r)$ - производная по $\ln r$ от $l(r)$. Ясно, что $k \in H$, причем, как хорошо известно (см., например, [3, с. 63]), $\rho(k)=+\infty$.

Положим $h(r)=(k(r))^{1 / 2}(r \geqslant 0)$. Тогда $h \in H, \rho(h)=+\infty$. Пусть, далее, $f-$ целая функция из теоремы 2 со свойствами 1)-3). Для нее соотношение (4), понятно, не выполняется. Легко видеть, что $f \in M(l)$. Действительно, так как $k(r) / \nu_{f}(r) \rightarrow+\infty$ $(r \rightarrow+\infty)$, а $\nu_{f}(r)$ - производная по $\ln r$ от $\ln \mu_{f}(r)$, то, воспользовавшись правилом Лопиталя, получаем $l(r) / \ln \mu_{f}(r) \rightarrow+\infty(r \rightarrow+\infty)$. Тогда в силу неравенства $T_{f}(r) \leqslant$ $\ln ^{+} M_{f}(r)$ и свойства 2) функции $f f \in M(l)$ и теорема 3 доказана.

$\mathbf{3}^{\circ}$. Докажем теорему 2 . Построение функции $f$ будем вести хорошо известньм методом, которым пользуется и Хейман [4] (см. также [3, с. 123-124]) для построения целой функции с исключительным множеством в соотношении (1). Идея оценки величины исключительного множества заимствована из работы автора [5].

Пусть $h \in H, \rho(h)=+\infty$. Тогда, очевидно, существует последовательность действительных чисел $\left\{c_{n}\right\}$ такая, что $c_{0} \geqslant 2, c_{n+1} \geqslant 2 c_{n}$ при $n \geqslant 0, h\left(c_{0}\right) \geqslant 2, \ln ^{-1} c_{n+1} \times$ $\ln \left[h\left(c_{n+1}\right)\right] \geqslant\left[h\left(c_{n}\right)\right]+1$ при $n \geqslant 0$. Рассматривая последовательность $\left\{\lambda_{n}\right\}$, для которой $\lambda_{0}=0$ и $\lambda_{n}=\left[h\left(c_{n-1}\right)\right]$ при $n \geqslant 1$, получаем, что

$$
\lambda_{n+1} \geqslant c_{n}^{\lambda_{n}+1} \geqslant 2 \lambda_{n}
$$

Пусть $a_{0}=1$ и $a_{n}=\left(c_{0}^{\lambda_{1}-\lambda_{0}} c_{1}^{\lambda_{2}-\lambda_{1}} \cdots c_{n-1}^{\lambda_{n}-\lambda_{n-1}}\right)^{-1}$ при $n \geqslant 1$. Тогда, воспользовавшись неравенствами (5), имеем $a_{n} \leqslant c_{n-1}^{-\left(\lambda_{n}-\lambda_{n-1}\right)} \leqslant c_{n-1}^{-\lambda_{n} / 2}$. Следовательно, функция $f(z)=\sum_{n=0}^{+\infty} a_{n} z^{\lambda_{n}}$ - целая. Докажем, что эта функция и является искомой.

Действительно, так как $a_{n} / a_{n+1}=c_{n}^{\lambda_{n+1}-\lambda_{n}}$ при $n \geqslant 0$, то непосредственно легко проверить (или см., например, [6]), что $\mu_{f}(r)=a_{n} r^{\lambda_{n}}$ и $\nu_{f}(r)=\lambda_{n}$ для всех $r \in\left[c_{n-1} ; c_{n}\right)$ при любом $n \geqslant 1$. Отсюда получаем $\nu_{f}(r) \leqslant h(r)\left(r \geqslant c_{0}\right)$, т. е. свойство 1) функции $f$ установлено. 
Далее, при $n \rightarrow+\infty$ имеем

$$
\begin{aligned}
\left(\sum_{k=0}^{n-1}+\sum_{k=n+2}^{+\infty}\right) \lambda_{k} a_{k} c_{n}^{\lambda_{k}}= & \mu_{f}\left(c_{n}\right)\left(\sum_{k=0}^{n-1} \lambda_{k} \frac{c_{k}^{\lambda_{k+1}-\lambda_{k}} c_{k+1}^{\lambda_{k+2}-\lambda_{k+1}} \cdots c_{n-1}^{\lambda_{n}-\lambda_{n-1}}}{c_{n}^{\lambda_{n}-\lambda_{k}}}\right. \\
& \left.+\sum_{k=n+2}^{+\infty} \lambda_{k} \frac{c_{n}^{\lambda_{k}-\lambda_{n+1}}}{c_{k-1}^{\lambda_{k}-\lambda_{k-1}} c_{k-2}^{\lambda_{k-1}-\lambda_{k-2}} \cdots c_{n+1}^{\lambda_{n+2}-\lambda_{n+1}}}\right) \\
\leqslant & \mu_{f}\left(c_{n}\right)\left(\sum_{k=0}^{n-1} \lambda_{k}\left(\frac{1}{2}\right)^{\lambda_{n}-\lambda_{k}}+\sum_{k=n+2}^{+\infty} \lambda_{k}\left(\frac{1}{2}\right)^{\lambda_{k}-\lambda_{n+1}}\right) \\
= & o\left(\mu_{f}\left(c_{n}\right)\right) .
\end{aligned}
$$

Отсюда следует, что $M_{f}\left(c_{n}\right)=(2+o(1)) \mu_{f}\left(c_{n}\right)$ при $n \rightarrow+\infty$. Так как $\ln M_{f}(r)$ и $\ln \mu_{f}(r)$ - соответственно вьпуклая и линейная функции относительно $\ln r$ на отрезке $\left[c_{n} ; c_{n+1}\right]$, то максимум разности $\ln M_{f}(r)-\ln \mu_{f}(r)$ (а, следовательно, и частного $\left.M_{f}(r) / \mu_{f}(r)\right)$ на этом отрезке достигается в одной из точек $c_{n}$ или $c_{n+1}$. Поэтому

$$
M_{f}(r)=(2+o(1)) \mu_{f}(r) \quad(r \rightarrow+\infty)
$$

и тем самым свойство 2) функции $f$ доказано.

Перейдем к доказательству свойства 3$)$. При $n \rightarrow+\infty$ равномерно по $\varphi \in[0 ; 2 \pi)$, согласно неравенствам (5) выполняется следуюшая цепочка неравенств:

$$
\begin{aligned}
\left|f^{\prime}\left(c_{n} e^{i \varphi}\right)\right| & \geqslant \lambda_{n+1} a_{n+1} c_{n}^{\lambda_{n+1}-1} \lambda_{n} a_{n} c_{n}^{\lambda_{n}-1}-\left(\sum_{k=0}^{n-1}+\sum_{k=n+2}^{+\infty}\right) \lambda_{k} a_{k} c_{n}^{\lambda_{k}} \\
& \geqslant\left(\frac{1}{2}-o(1)\right) \mu_{f}^{2}\left(c_{n}\right) .
\end{aligned}
$$

Учитьвая неравенство (6), отсюда получаем

$$
\varlimsup_{r \rightarrow+\infty} \frac{T_{f^{\prime}}(r)}{T_{f}(r)} \geqslant \varlimsup_{r \rightarrow+\infty} \frac{T_{f^{\prime}}(r)}{\ln \mu_{f}(r)} \geqslant 2 .
$$

С другой стороны, из (3) и леммы о логарифмической производной следует, что

$$
\varliminf_{r \rightarrow+\infty} \frac{T_{f^{\prime}}(r)}{T_{f}(r)} \leqslant 1 .
$$

Зафиксируем, далее, любые $\varepsilon, \delta$ и $\alpha$, удовлетворяюшие неравенствам $0<\varepsilon<\delta<$ $\alpha<1$. Так как $T_{f}(r) \leqslant(1+\delta)(1+\varepsilon)^{-1} \ln \mu_{f}(r)$ при $r \geqslant r_{2} \geqslant c_{0}$, то $E_{3}(\delta, f)=\left\{r>r_{2}\right.$ : $\left.T_{f^{\prime}}(r)>(1+\delta) \ln \mu_{f}(r)\right\}$ - подмножество множества $E_{1}(\varepsilon, f)$. Отметим, что множество $E_{3}(\delta, f)$ является открытым, в связи с чем его можно представить в виде объединения счетного числа взаимно непересекающихся интервалов. Выберем из этого объединения бесконечную последовательность $\left\{\left(x_{n} ; y_{n}\right)\right\}$ интервалов, для которых при любом $n \geqslant 0$ выполнены условия:

1) $x_{n}<y_{n}<x_{n+1}$

2) $T_{f^{\prime}}\left(t_{n}\right)=(1+\alpha) \ln \mu_{f}\left(t_{n}\right)$ для некоторой точки $t_{n} \in\left(x_{n} ; y_{n}\right)$. 
Ясно, что $T_{f^{\prime}}\left(x_{n}\right)=(1+\delta) \ln \mu_{f}\left(x_{n}\right), T_{f^{\prime}}\left(y_{n}\right)=(1+\delta) \ln \mu_{f}\left(y_{n}\right)$. Поэтому

$$
\ln \mu_{f}\left(y_{n}\right)-\ln \mu_{f}\left(x_{n}\right)>\frac{T_{f^{\prime}}\left(y_{n}\right)}{1+\delta}-\frac{T_{f^{\prime}}\left(t_{n}\right)}{1+\alpha}>(\alpha-\delta) T_{f^{\prime}}\left(y_{n}\right) \underset{n \rightarrow+\infty}{\rightarrow}+\infty
$$

Следовательно,

$$
\int_{E_{1}(\varepsilon, f)} h(r) d r \geqslant \int_{\cup_{n=0}^{+\infty}\left(x_{n} ; y_{n}\right)} \frac{\nu_{f}(r)}{r} d r=\int_{\cup_{n=0}^{+\infty}\left(x_{n} ; y_{n}\right)} d \ln \mu_{f}(r)=+\infty
$$

и теорема 2 доказана.

\section{СПИСОК ЦИТИРОВАННОЙ ЛИТЕРАТУРЫ}

[1] Hayman W. K. The local growth of power series; a survey of the Wiman-Valiron method // Canad. Math. Bull. 1974. V. 17. № 3. P. 317-358.

[2] Виттих Г. В. Новейшие исследования по однозначным аналитическим функциям. М.: Физматгиз, 1960.

[3] Гольдберг А. А., Островский И.В. Распределение значений мероморфных функций. М.: Наука, 1970.

[4] Hayman W.K. On the characteristic of functions meromorphic in the plane and of their integrals // Proc. London Math. Soc. Ser. A. 1965. V. 14. P. 93-128.

[5] Філевич П. В. До теореми Лондона про співвідношення Бореля для цілих функцій // Укр. матем. ж. 1998. Т. 50. №11. С. 1578-1580.

[6] Шеремета М.Н. Об эквивалентности логарифмов максимума модуля и максимального члена целого ряда Дирихле // Матем. заметки. 1987. Т. 42. №2. С. 215-226.

Львовский государственньй университет им. И. Франко 